\title{
Dynamic Signatures of Driven Vortex Motion
}

\author{
G.W. Crabtree, ${ }^{1}$ D. Lopez, ${ }^{1} *$ W.K. Kwok, ${ }^{1}$ A.M. Petrean, ${ }^{1,2}$ \\ R.J. Olsson, ${ }^{1,3}$ H. Safar, ${ }^{4, *}$ and L.M. Paulius ${ }^{1,2}$
}

'Materials Science Division, Argonne National Laboratory, Argonne, IL 60439 USA

${ }^{2}$ Department of Physics, Western Michigan University, Kalamazoo, MI 49008 USA

${ }^{3}$ Department of Physics, Michigan State University, East Lansing, MI 48824 USA

${ }^{4}$ Department of Physics, University of Illinois at Chicago, Chicago, IL 60680 USA

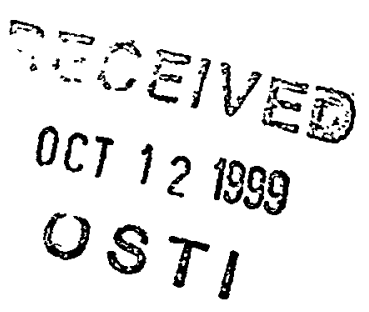

To be Published in the Proceedings of the Second Polish-US Conference on High Temperature Superconductivity Wroclam and Kudowa, August 17-21, 1998.

This work was supported by the U.S. Department of Energy, Office of Basic Energy SciencesMaterials Sciences, under contract \#W-31-109-ENG-38 (GWC, WKK) and the NSF Science and Technology Center for Superconductivity under contract \#DMR91-20000 (DL). 


\section{DISCLAIMER}

This report was prepared as an account of work sponsored by an agency of the United States Government. Neither the United States Government nor any agency thereof, nor any of their employees, make any warranty, express or implied, or assumes any legal liability or responsibility for the accuracy, completeness, or usefulness of any information, apparatus, product, or process disclosed, or represents that its use would not infringe privately owned rights. Reference herein to any specific commercial product, process, or service by trade name, trademark, manufacturer, or otherwise does not necessarily constitute or imply its endorsement, recommendation, or favoring by the United States Government or any agency thereof. The views and opinions of authors expressed herein do not necessarily state or reflect those of the United States Government or any agency thereof. 


\section{DISCLAIMER}

Portions of this document may be illegible in electronic image products. Images are produced from the best available original document. 


\title{
Dynamic Signatures of Driven Vortex Motion
}

\author{
G. W. Crabtree, ${ }^{1}$ D. Lopez, ${ }^{1, *}$ W. K. Kwok, ${ }^{1}$ A. M. Petrean, ${ }^{1,2}$ \\ R. J. Olsson, ${ }^{1,3}$ H. Safar, ${ }^{4,}{ }^{*}$ and L. M. Paulius. 1,2

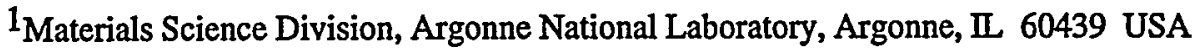 \\ ${ }^{2}$ Department of Physics, Western Michigan University, Kalamazoo, MI 49008 USA \\ ${ }^{3}$ Department of Physics, Michigan State University, East Lansing, MI 48824 USA \\ ${ }^{4}$ Department of Physics, University of Illinois at Chicago, Chicago, II 60680 USA
}

\begin{abstract}
We probe the dynamic nature of driven vortex motion in superconductors with a new type of transport experiment. An inhomogeneous Lorentz driving force is applied to the sample, inducing vortex velocity gradients that distinguish the hydrodynamic motion of the vortex liquid from the elastic and-plastic motion of the vortex solid. We observe elastic depinning of the vortex lattice at the critical current, and shear induced plastic slip of the lattice at high Lorentz force gradients.
\end{abstract}




\section{Introduction}

The melting of the vortex solid to a liquid is one of the most basic phenomena in high temperature superconductivity. This fundamental phase change from order to disorder induces novel behavior in the structural, electrodynamic, and thermal properties of superconductors. The phase transition itself has many fascinating aspects, including first and second order thermodynamic character, upper and lower critical points, an associated peak effect in the critical current, strong angular dependence, and sensitivity to pinning disorder. (For reviews, see Refs. 1-5.) Strong interest in the nature of melting has stimulated creative experiments on the resistivity, ${ }^{6-12}$ magnetization, ${ }^{13-17}$ and heat capacity ${ }^{18-23}$ at the transition. These experiments provide valuable information on the thermodynamic properties of melting, and on the dramatic differences in pinning strengths and critical currents of the solid and liquid vortex phases.

There are equally interesting dynamic properties associated with the vortex phases. In the liquid state, vortices are free to move past each other in response to variations in the driving and pinning forces they experience. The resulting motion is hydrodynamic, where the velocity profile changes continuously on the scale of the intervortex distance. Hydrodynamic motion can be described by differential equations which provide a complete picture of the velocity distribution if the driving force and boundary conditions are known. ${ }^{24-26}$ In the solid vortex states, however, elastic bonds between vortices prevent vortices from moving past each other. ${ }^{27}$ If the bonds do not break, the vortex system moves as a single elastic object with one average velocity for all vortices. If shear forces are large enough to break the elastic bonds, planes or surfaces of plastic slip are introduced where the velocity profile changes abruptly. The elastic character of the moving solid is the fundamental feature distinguishing it from the hydrodynamic motion of the liquid. The elastic shear modulus of the solid resists an applied shear stress by constraining neighboring vortices to move at the same velocity, while the liquid accommodates an applied shear by allowing neighboring vortices to move at different velocities.

Conventional transport experiments where the vortex system is driven with a spatially uniform Lorentz force are only weakly sensitive to the differences between liquid and solid motion. In the absence of pinning, the liquid and solid move with uniform velocity and their differing response to shear forces plays no role in the motion. Pinning disorder introduces shear forces and velocity profiles that in principle could distinguish liquids from solids. However, the velocity differences are averaged out on the scale of the pinning disorder, typically well below the spatial resolution of transport experiments.

In this article, we describe a new class of transport experiments where the vortex system is driven with an inhomogeneous Lorentz force. ${ }^{28}$ The response of the vortex system to the gradient in the applied driving force reveals the fundamental signatures of liquid and solid motion. We demonstrate a qualitative change in the character of the motion at the vortex melting transition, and we show explicitly that the moving vortex lattice maintains dynamic coherence over distances of order the sample size. We use a high driving force gradient to introduce plastic slip surfaces into the moving lattice and we see the number of slip surfaces increase with the strength of the applied shear stress. 


\section{Controlled Gradient Transport}

Our experiments are carried out in the disk geometry illustrated in the inset of Figure 1. Current is injected at the center of the disk and removed at the circumference through gold electrodes evaporated onto the sample. In this geometry the current flows in the radial direction, with inhomogeneous magnitude $J(r)=I /(2 \pi r t)$, where $t$ is the sample thickness. The Lorentz force due to this current drives vortices azimuthally in circular orbits around the disk. Vortices in these orbits never encounter the sample surface, ensuring that the measured transport properties reflect only bulk vortex behavior unaffected by entry and exit effects, Bean Livingston barriers, or surface pinning. The inhomogeneous current distribution produces a Lorentz force gradient varying as $1 / \mathrm{r}^{2}$. This gradient introduces a spatially varying shear stress which probes the dynamic response of the liquid and solid phases.

Our experiments were carried out on untwinned $\mathrm{YBa}_{2} \mathrm{Cu}_{3} \mathrm{O}_{\mathrm{x}}$ crystals carefully polished into disk geometries, of diameter approximately $700 \mu \mathrm{m}$ and thickness $10 \mu \mathrm{m}$. Voltage leads were placed along a radius of the sample at intervals of approximately $60 \mu \mathrm{m}$ from the center current electrode and from each other, as indicated in the inset of Figure 1. Transport measurements were made with standard ac and dc methods. Further details of the experiment may be found in Ref. 28.

\section{Normal Metallic State}

The upper panel of Figure 1 shows the voltage measured between pairs of voltage leads as a function of temperature at zero field in the normal state below $240 \mathrm{~K}$. The voltages decrease with increasing radius, reflecting the decreasing current density in the disk geometry. In the normal state with Ohmic resistivity $\rho=E / J$, the voltage as a function of radius is given by

$$
V_{n, n+1}=\int_{r_{n}}^{r_{n+1}} E(r) d r=\frac{\rho I}{2 \pi t} \ln \left(\frac{r_{n+1}}{r_{n}}\right)
$$

The voltages scaled by the logarithmic term on the right should collapse onto a single line characteristic of the resistivity. The lower panel of Figure 1 shows the scaled voltages. Their collapse to a single line confirms that the current density falls off like $1 / \mathrm{r}$ as expected for the disk geometry.

\section{Vortex Liquid State}

In the superconducting state in finite field, the transport voltage arises from the motion of vortices driven by the Lorentz force. Here the electric field is not directly related to the current density as in the normal state. Instead, the electric field is governed by the vortex velocity through

$$
\mathbf{E}=\mathbf{B} \mathbf{X} \mathbf{v} / \mathbf{c}
$$


The vortex velocity, in turn, is determined by the balance between the Lorentz driving force and the pinning forces opposing the motion. In the liquid state, the velocity is given by the solution to the hydrodynamic equation

$$
-\gamma \mathbf{v}+\eta \nabla_{\perp}^{2} \mathbf{v}+\mathrm{F}_{\mathrm{L}}=0
$$

where $\eta$ is the shear viscosity and $\gamma$ the dynamic friction opposing the vortex motion. The solution of the hydrodynamic equation for the disk geometry is given by Marchetti and Nelson. ${ }^{26}$ They discuss a method of measuring the dynamic correlation length from the velocity profile in the vicinity of a no-slip boundary condition as might be imposed by the sample edge or an extended pinning structure. Here we consider the free response of the vortex liquid far from any boundary conditions. In this region, a $1 / r$ driving current induces a $1 / r$ velocity profile. Physically this means that the velocity of the liquid follows the local driving force. The liquid fully accommodates the applied shear force by adjusting its velocity accordingly. This fundamental property of hydrodynamic motion can be directly observed in our experiment.

The electric field induced by the vortex motion in the liquid is $E(r)=B v(r) / c=\left(B \phi / \gamma c^{2}\right) J(r)$ $=\rho_{\mathrm{f}} \mathrm{J}(\mathrm{r})$, where $\rho_{\mathrm{f}}$ is the flux flow resistivity. This is the same form as for the normal state, with the Ohmic resistivity replaced by the flux flow resistivity. Thus hydrodynamic scaling follows Eq. (1) with $\rho$ replaced by $\rho_{f}$.

The upper panel of Figure 2 shows the measured voltages in the vortex liquid state, as a function of temperature for applied fields of zero and $4 \mathrm{~T}$. The voltages decrease with radius, indicating that the vortices circulate at lower velocity as the radius increases. The lower panel of Figure 2 shows the same voltages scaled according to Eq. (1) and expressed as flux flow resistivity. The three curves collapse to a single curve, demonstrating the long range $1 / \mathrm{r}$ velocity profile characteristic of hydrodynamic motion of the liquid.

\section{Vortex Lattice State}

In the vortex lattice, the shear viscosity of the liquid is replaced by shear elasticity. The elastic bonds between neighboring vortices require them to move with the same velocity as long as the bonds remain intact. Velocity changes can occur, however, by plastic shear where the elastic bonds between neighboring vortices are broken. Thus the smooth variation of the velocity on the scale of intervortex distances is eliminated and the motion is no longer hydrodynamic. The change from hydrodynamic flow to solid-like flow appears in the experiment as a failure of the hydrodynamic scaling. This is shown in the upper panel of Figure 3, where the measured voltages are shown in the vicinity of the melting point. Just below the melting point $T_{M}$ there is a break in the curves (marked by an arrow in Figure 3 ) indicating a slowdown in the vortex velocity as the shear elasticity of the lattice enhances its pinning effectiveness. Below this break the three curves begin to approach each other, eventually crossing in pairs. At lower temperatures, the order of the curves is reversed, with the outer vortices traveling faster than the inner ones. This order is opposite to that of the liquid phase and it cannot be described by hydrodynamic motion. 
The nature of the motion in the lattice phase is revealed in the lower panel of Figure 3 . Here the measured voltages are scaled according to elastic rotation. If the lattice rotates elastically, the velocity of any vortex is linearly proportional to its radius and the rate of rotation, $v(r)=\omega r$. Then the electric field is $E(r)=B \omega r / c$, and the voltage between taps is given by

$$
V_{n, n+1}=\int_{r_{n}}^{r_{n+1}} E(r) d r=\frac{B \omega}{2 c}\left(r_{n+1}^{2}-r_{n}^{2}\right)
$$

The lower panel of Figure 3 shows the voltages scaled by Eq. (4) and plotted as the angular velocity $\omega$. At the break in the curves just below $T_{M}$, the angular velocity of the lattice sampled by the three sets of voltage taps begins to equalize. This marks the termination of hydrodynamic flow with its $1 / \mathrm{r}$ velocity scaling, and the onset of plastic flow. At lower temperature, the three curves collapse to a single curve. Here the lattice rotates elastically with a single angular velocity. The degree of dynamic correlation in this regime is remarkable- the dynamic correlation length exceeds the distance between the innermost and outermost voltage taps. The velocity correlation is macroscopic, of order the size of the sample.

In the temperature range between the hydrodynamic and elastic motion, there is an interesting region of plastic motion reflecting the influence of the shear elasticity of the lattice. Here there is a competition between the shear modulus of the lattice favoring a constant angular velocity and the shear component of the Lorentz force favoring faster rotation of the inner vortices over the outer ones. Neither has sufficient strength to dominate the other, with the result that the lattice moves at short range in elastic flow interrupted by plastic slip surfaces which relieve the applied shear stress over large distances. As the temperature decreases and the rotation slows down, the shear elasticity of the lattice gradually overcomes the applied shear stress, and the dynamic correlation length grows until the entire lattice rotates as a single elastic object.

The nature of plastic motion in the rotating lattice can be seen by following the rotational velocities as measured by the inner, center, and outer sets of voltage taps as the driving shear stress is increased. Figure 4 shows I-V curves for the three pairs of voltage taps plotted as angular velocity at two temperatures in an applied magnetic field of $5 \mathrm{~T}$. Below the critical current, the lattice is stationary. At the critical current, vortices depin and begin to move in circular orbits. The depinning process itself is interesting in this crystal. Figure 4 shows that the lattice rotates elastically immediately on depinning, as indicated by the single angular velocity sampled by all three pairs of voltage taps. Conventional transport experiments cannot distinguish between this kind of elastic depinning and the more familiar plastic depinning where the weakly pinned vortices begin to move while the strongly pinned ones remain at rest.

In elastic rotation the lattice is under shear stress since the driving force distribution varying as $1 / r$ is out of balance with the velocity distribution varying as $r$. This shear stress is accommodated by elastic distortions in the rotating lattice. As the driving current increases, the shear stress also increases, until it exceeds the elastic limit of the shear modulus. At this point the shear stress is relieved by plastic slip in the lattice at a fixed radius, and the entire lattice no longer rotates with a single angular velocity. This effect can be seen in Figure 4, where the angular velocities sampled by the three voltage taps separate as indicated by arrows. The inner section of the lattice is the first to shear, as the angular velocity sampled by the inner taps rises 
above the others. The faster rotation of the inner section is consistent with the larger driving force there, and it partially relieves the shear stress in the rotating lattice. At this point plastic slip has occurred only in the innermost section, with the outer two sections rotating elastically with a common angular velocity. As the gradient of the driving force continues to increase, plastic slip interrupts the coherent motion of the center and outermost sections, as indicated by the second arrow in Figure 4. These data reveal the onset and evolution of plastic motion under increasing shear stress. The lattice separates into concentric rings, each rotating with successively slower angular velocity as the radius increases. As the shear stress increases, more fault lines are introduced into the lattice, reducing the radial dynamic correlation length at each step.

The transformation of elastic flow to plastic flow by the introduction of slip lines can be visualized on the driving force-temperature phase diagram of Figure 5. Here the regions of pinned solid, elastic rotation, and shear induced slip at $5 \mathrm{~T}$ as a function of temperature and driving force are shown. The critical current as a function of temperature defines the boundary between the pinned solid and elastic rotation. At higher driving force the first observable shear induced slip occurs between the inner set of taps and the center and outer sets of taps, indicated by the first arrow in Figure 4. The middle line in Figure 5 shows the temperatures and driving forces where this slip occurs at $5 \mathrm{~T}$. The highest lying line indicates the onset of slip between the center and outer sets of voltage taps. The critical currents for depinning and the onset of plastic shear all approach zero at the melting temperature. Here the shear modulus of the lattice disappears, the motion becomes hydrodynamic, and the velocity adjusts to the driving force gradient on the intervortex length scale.

One interesting question is the nature of the transition from solid to liquid in the presence of strong shear induced slip. As the applied shear stress increases the lattice adjusts by increasing the radial density of plastic slip lines. The physical limit for this process is one slip line per radial intervortex spacing, allowing velocity changes on the same length scale as in hydrodynamic motion. The difference betwieen this high density plastic slip and hydrodynamic flow is an interesting basic question.

\section{Summary}

We describe a new type of transport experiment where an inhomogeneous current density is applied to the sample to produce a gradient in the Lorentz driving force. The vortex velocity profiles induced by the driving force gradient reveal fundamental features of the dynamics of the driven motion. The measured velocity profiles distinguish hydrodynamic motion of the vortex liquid from elastic and plastic motion of the solid. Elastic depinning of the vortex lattice at the critical current is observed, implying that the elastic bonds between vortices dominate the random pinning forces in our crystal. By controlling the driving force and its gradient, we regulate the number of shear-induced plastic slip lines in the moving lattice. The various pinned, plastic, and elastic dynamic states of the vortex lattice and hydrodynamic flow of the liquid are visualized on a driving force-temperature phase diagram.

The controlled gradient transport experiment described above offers new opportunities to explore the basic features of the vortex phases in superconductors. We have examined the responses of 
the vortex liquid and lattice to a gradient in the driving force, finding characteristic signatures of the underlying viscous or elastic natures of these phases. The response of the disordered vortex states such as the vortex and Bose glasses, and of vortex liquid states that are strongly pinned or entangled promises new insights into their fundamental static and dynamic character.

This work was supported by the US Department of Energy, Office of Basic Energy SciencesMaterials Sciences, under contract \#W-31-ENG-38 (GWC, WKK) and the NSF Science and Technology Center for Superconductivity under contract \#DMR91-20000 (DL). 


\section{References}

* Present Address: Lucent Technologies, Murray Hill, NJ 07964 USA

1. Crabtree, G. W., Kwok, W. K., Welp, U. Lopez, D., and Fendrich, J. A. Vortex Melting and the Liquid State in $\mathrm{YBa}_{2} \mathrm{Cu}_{3} \mathrm{O}_{\mathrm{x}}$, in Physics and Materials Science of Vortex States, Flux Pinning, and Dynamics, Proceedings of the NATO ASI in Kusadasi, Turkey, 1998, (R. Kossowsky, S. Bose, V. Pan, and Z. Durusoy, eds) Kluwer (Dordrecht), 1999, p. 357.

2. Crabtree, George W. and Nelson, David R. (1997) Vortex Physics in High Temperature Superconductors, Physics Today 50 (4), 38-45.

3. Crabtree, G. W., Welp, U., Kwok, W. K., Fendrich, J. A., and Veal, B. W. (1997) The equilibrium vortex melting transition in $\mathrm{YBa}_{2} \mathrm{Cu}_{3} \mathrm{O}_{7}$, J. Alloys and Compounds 250, 609614.

4. Crabtree, G. W., Kwok, W. K., Welp, U., Fendrich, J. A., and Veal, B. W. (1996) Static and Dynamic Vortex Transitions in Clean YBa2Cu3O7, J. Low Temp. Phys. 105, 1073-1082.

5. Blatter, G., Feigel'man, M. V., Geshkenbein, V. B., Larkin, A. I., and Vinokur, V. M. (1994) Vortices in high temperature superconductors, Rev. Mod. Phys. 66, 1125-1388.

6. Safar, H., Gammel, P. L., Huse, D. A., Bishop, D. J., Rice, J. P., Ginsberg, D. M. (1992) Experimental evidence for a first order vortex lattice melting transition in untwinned, single crystal YBCO, Phys. Rev. Lett. 69, 824-828.

7. Kwok, W. K., Fleshler, S., Welp, U., Vinokur, V. M., Downey, J., Crabtree, G. W., Miller, M. M.,(1992) Vortex lattice melting in untwinned and twinned single crystals of YBCO, Phys. Rev. Lett. 69, 3370.

8. Safar, H. Gammel, P. L., Huse, D. A., Bishop, D. J., Lee, W. C., Giapintzakis, J., Ginsberg, D. M. (1993) Experimental evidence for a multicritical point in the magnetic phase diagram for the mixed state of clean, untwinned YBCO, Phys. Rev. Lett. 70, 3800-3803.

9. Kwok, W. K., Fendrich, J., Fleshler, S., Welp, U., Downey, J., and Crabtree, G. W. (1994) Vortex liquid disorder and the first order melting transition in YBCO, Phys. Rev. Lett. 72, 1092-1096.

10. Fendrich, J. A., Kwok, W. K., Giapintzakis, J., van der Beek, C. J., Vinokur, V. M., Fleshler, S., Welp, U., Viswanathan, H. K., Crabtree, G. W. (1995) Vortex liquid state in an electron irradiated untwinned YBCO crystal, Phys. Rev. Lett. 74, 1210-1214.

11. Lopez, D., Krusin-Elbaum, L., Safar, H., Righi, E., de la Cruz, F., Grigera, S., Feild, C., Kwok, W. K., Paulius, L., and Crabtree, G. W. (1998) Pinned vortex liquid above the critical point of the first order melting transition: a consequence of point-like disorder, Phys. Rev. Lett. 80, 1070-1073. 
12. W. K. Kwok, Fendrich, J. A., Vinokur, V. M., Koshelev, A. E., and Crabtree, G. W. (1996) Vortex shear modulus and lattice melting in twin boundary channels of $\mathrm{YBa}_{2} \mathrm{Cu}_{3} \mathrm{O}_{\mathrm{x}}$, Phys. Rev. Lett. 76, 4596.

13. Pastoriza, H., Goffman, M. F., Arribére, A., and de la Cruz, F., (1994) First order phase transition at the irreversibility line of $\mathrm{Bi}_{2} \mathrm{Sr}_{2} \mathrm{CaCl}_{2} \mathrm{O}_{8}$, Phys. Rev. Lett. 72, 2951-2954.

14. Zeldov, E., Majer, D., Konczykowski, M., Geshkenbein, V. B., Vinokur, V. M. (1995) Thermodynamic observation of first order vortex-lattice melting transition in BSCCO, Nature (London) 375, 373-376.

15. Liang, R., Bonn, D. A., and Hardy, W. (1996) Discontinuity of reversible magnetization in untwinned YBCO single crystals at the first order vortex melting transition, Phys. Rev. Lett. 76, 835-839.

16. Welp, U., Fendrich, J. A., Kwok, W. K., Crabtree, G. W., and Veal, B. W. (1996) Thermodynamic evidence for a flux line lattice melting transition in YBCO, Phys. Rev. Lett. 76, 4809-4013.

17. Fendrich, J. A., Welp, U., Kwok, W. K., Koshelev, A. E., Crabtree, G. W., and Veal, B. W. (1996) Static and dynamic vortex phases in YBCO, Phys. Rev. Lett. 77, 2073-2077.

18. Schilling, A., Fisher, R. A., Phillips, N. E., Welp, U., Kwok, W. K., and Crabtree, G. W. (1997) Anisotropic latent heat of vortex-lattice melting in untwinned YBCO, Phys. Rev. Lett. 78, 4833-4838.

19. Roulin, M,. Junod, A., Walker, E. (1998) Observation of second order transitions below $T_{c}$ in the specific heat of YBCO: case for the melting of a vortex glass, Physica C 296, 137-152.

20. Roulin, M., Junod, A., Erb, A., Walker, E. (1998) Calorimetric transitions in the melting line of the vortex system.as a function of oxygen deficiency in high purity YBCO, Phys. Rev. Lett. 80, 1722-1726.

21. Bouquet, F., Marcenat, C., Calemczuk, R., Erb, A., Junod, A., Roulin, M., Welp, U., Kwok, W. K., Crabtree, G. W., Phillips, N. E., Fisher, R. A., and Schilling, A. (1999) Calorimetric evidence of the transitions between the different vortex states in YBCO, in Physics and Materials Science of Vortex States, Flux Pinning, and Dynamics, Proceedings of the NATO ASI in Kusadasi, Turkey, 1998, (R. Kossowsky, S. Bose, V. Pan, and Z. Durusoy, eds) Kluwer (Dordrecht), 1999, p. 743.

22. Roulin, M., Revaz, B., Junod, A., Erb, A, and Walker, E., High resolution specific heat experiments on the vortex melting line in $\mathrm{MBa}_{2} \mathrm{Cu}_{3} \mathrm{O}_{\mathrm{x}}(\mathrm{M}=\mathrm{Y}, \mathrm{Dy}$, and $\mathrm{Eu})$ crystals: observation of first and second order transitions up to $16 \mathrm{~T}$ (1999) in Physics and Materials Science of Vortex States, Flux Pinning, and Dynamics, Proceedings of the NATO ASI in 
Kusadasi, Turkey, 1998, (R. Kossowsky, S. Bose, V. Pan, and Z. Durusoy, eds) Kluwer (Dordrecht), 1999, p. 489.

23. Revaz, B., Junod, A., and Erb, A. (1998) Specific heat peaks observed up to $16 \mathrm{~T}$ on the melting line of vortex matter in DyBCO Phys. Rev. $B$ 58,11153-11156.

24. Marchetti, M. C. and Nelson, D. R. (1990) Hydrodynamics of flux liquids, Phys. Rev. B 42, 9938.

25. Marchetti, M. C. and Nelson, D. R. (1991) Dynamics of flux line liquids in high-T $T_{c}$ superconductors, Physica $C$ 174, 40-62.

26. Marchetti, M. C. and Nelson, D. R. (1999) Patterned geometries and hydrodynamics at the yortex Bose glass transition, Phys Rev B 59, pp. 13624-13627.

27. Crabtree, G. W., Leaf, G. K., Kaper, H. G., Vinokur, V. M., Koshelev, A. E. , Braun, D. W., and Levine, D. M. (1996) Driven motion of vortices in superconductors, in Recent Developments in High Temperature Superconductivity (Proceedings of the First Polish-US Conference on Superconductors, Duszniki Zdroj, Poland, Sept 10-14, 1995), Lecture Notes on Physics Series, Springer-Verlag (J. Klamut, B. W. Veal, B. Dabrowski, and P. Klamut, eds) Berlin, 1996, p 303.

28. Lopez, D., Kwok, W. K., Safar, H., Olsson, R. J., Petrean, A. M., Paulius, L. and Crabtree, G. W. (1999) Spatially resolved dynamic correlation in the vortex state of high temperature superconductors, Phys. Rev. Lett. 82, 1277. 


\section{Figure Captions}

Figure 1. Upper panel: the measured voltages in the normal metallic state for the three sets of voltage taps indicated in the inset. Lower panel: The measured voltages scaled by Eq.(1) of the text. The collapse to a single curve verifies the $1 / \mathrm{r}$ dependence of the applied current density.

Figure 2. Upper panel: the measured voltages of the taps shown in the inset in the vortex liquid state as a function of temperature in a magnetic field of $4 \mathrm{~T}$. Lower panel: the measured voltages scaled according to $\mathrm{Eq}(1)$ of the text with the resistivity $\rho$ replaced by the flux flow resistivity $\rho_{\mathrm{f}}$. The scaling of the three curves is a characteristic feature of hydrodynamic flow.

Figure 3. Upper panel: measured voltages in the vortex lattice state for three pairs of taps indicated in the inset of the lower panel. Results for two transport currents are shown. 0.5 $\mathrm{mA}$ is below the critical current of the lattice, while $15 \mathrm{~mA}$ is above the critical current and drives the lattice into motion. $T_{M}$ indicates the melting temperature and the arrow indicates the loss of hydrodynamic motion on cooling. Lower panel: the measured voltages scaled according to Eq. (4) of the text and expressed as rotational velocity. The collapse to a single curve at low temperature indicates elastic rotation of the lattice with a single angular velocity.

Figure 4. I-V characteristics for the vortex lattice at the field and temperatures indicated. The solid line indicates the voltage between the inner taps, the filled circles that between the center taps, and the open squares that between the outer taps. Arrows indicate the onset of plastic slip.

Figure 5. Phase diagram showing regions of the pinned lattice, elastic rotation, plastic rotation, and hydrodynamic motion as a function of driving force and temperature. The middle and upper lines correspond the to the first and second arrows in Figure 4, respectively. 

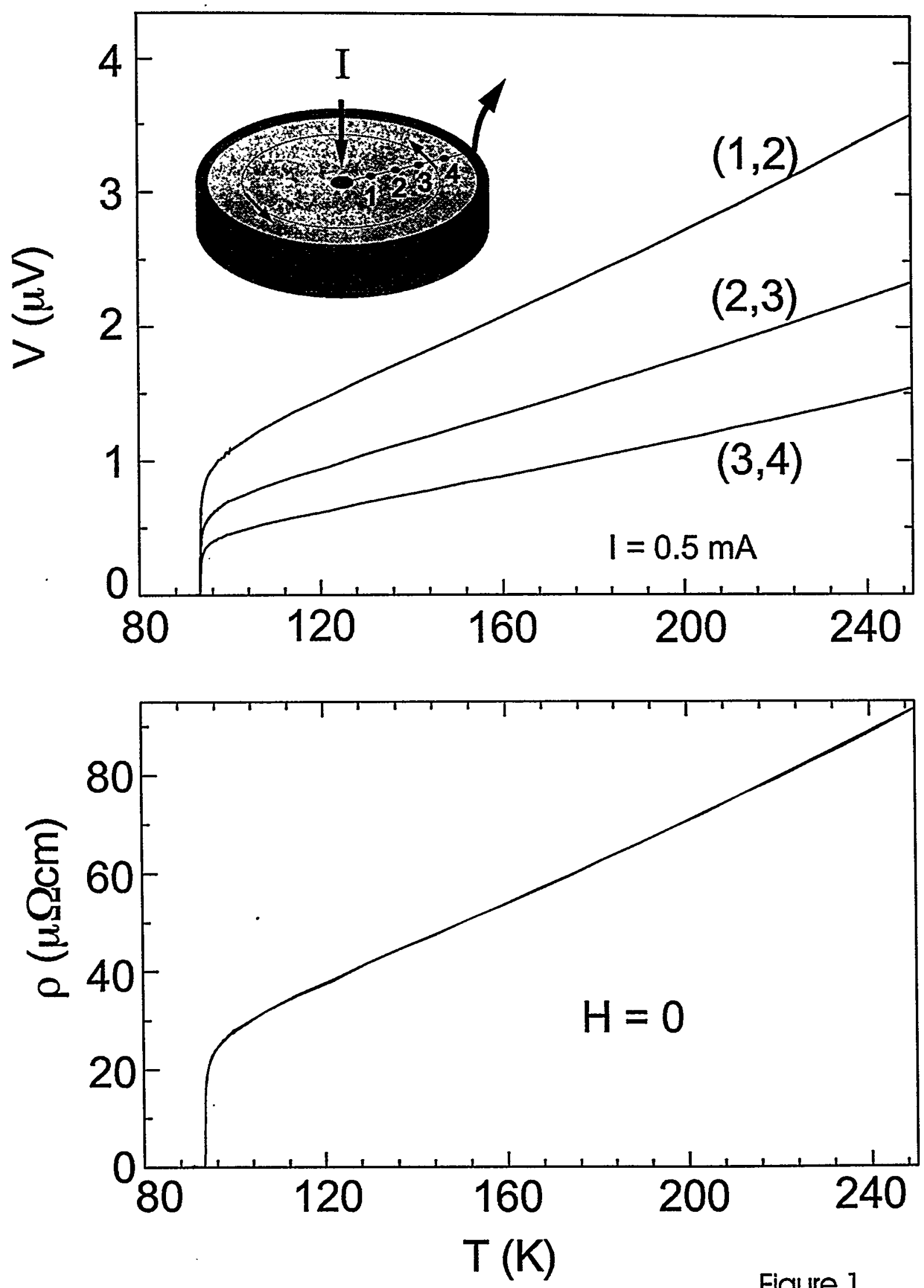

Figure 1

Crabtree et al 



Figure 2

Crabtree et al 

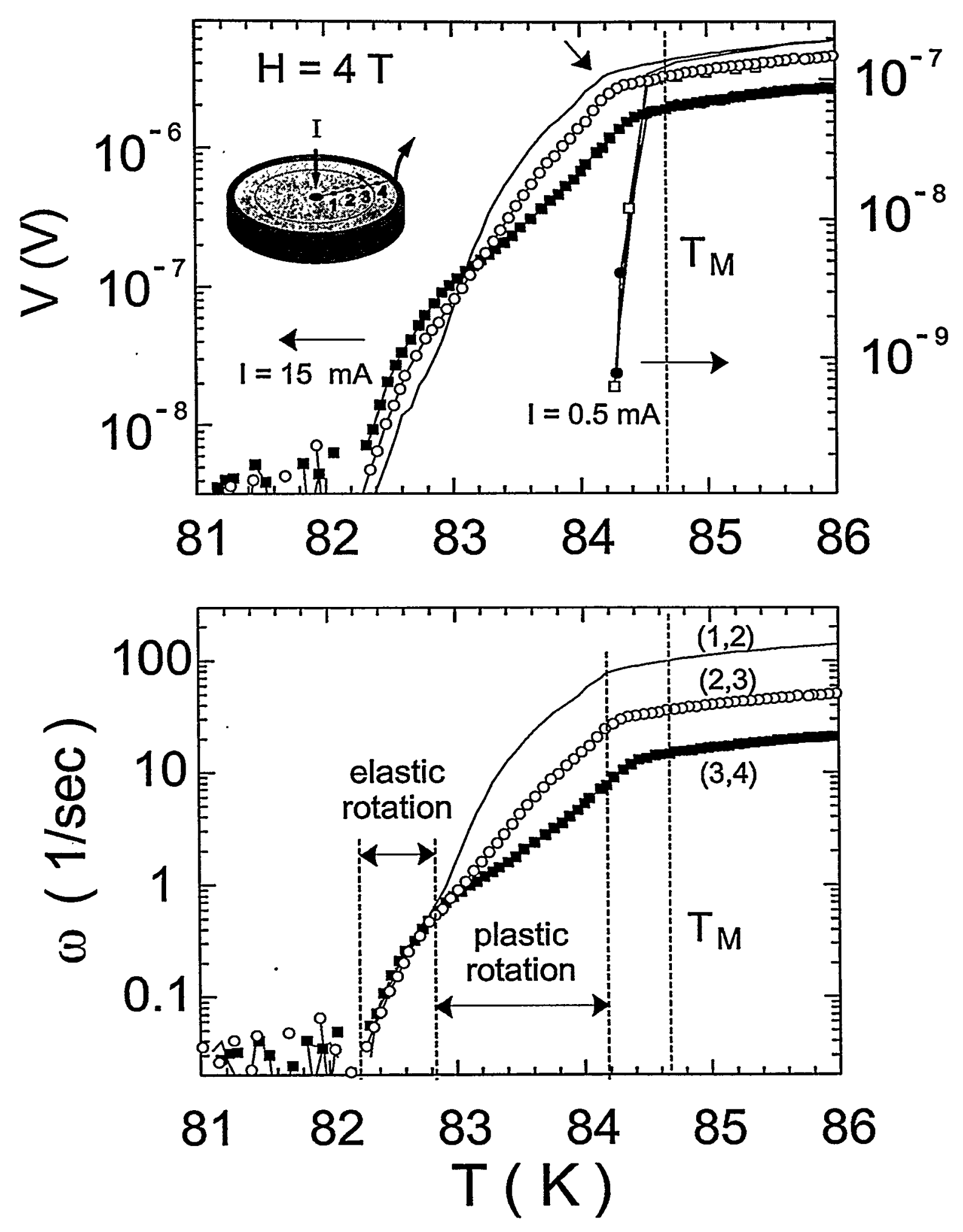

Figure 3 rev 8/1 1/99 Crabtree et al 


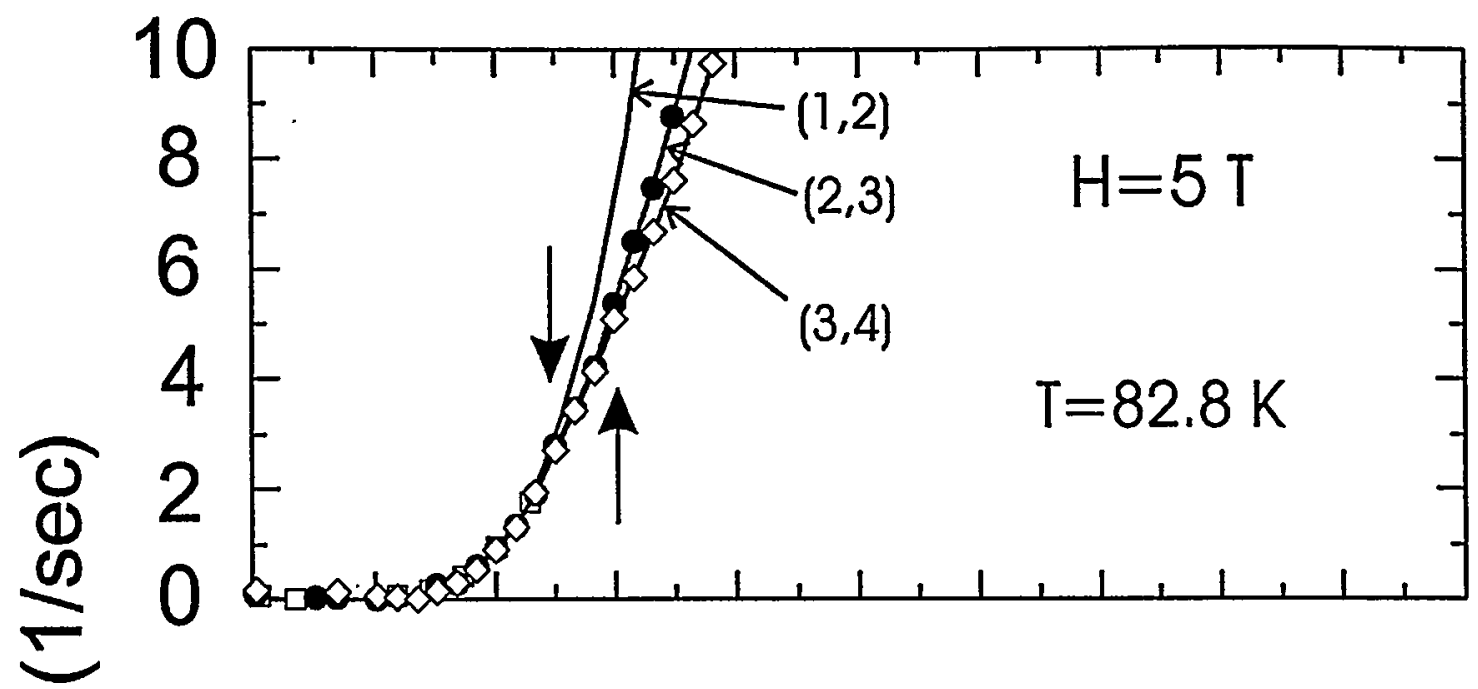

310
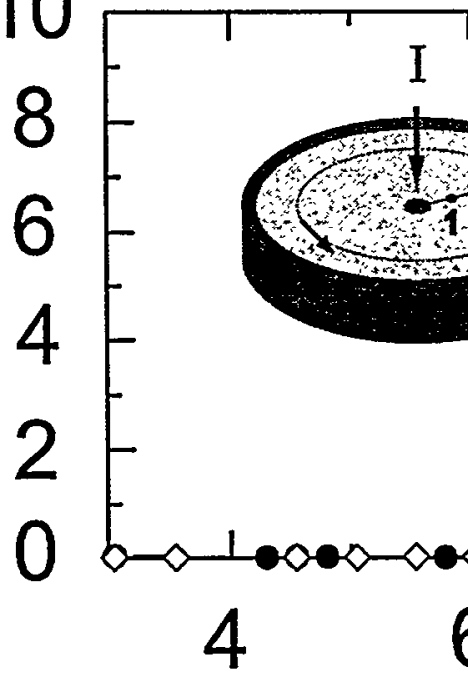

$(1,234$

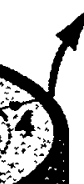

$3 \times+2$

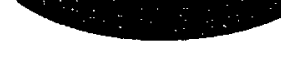




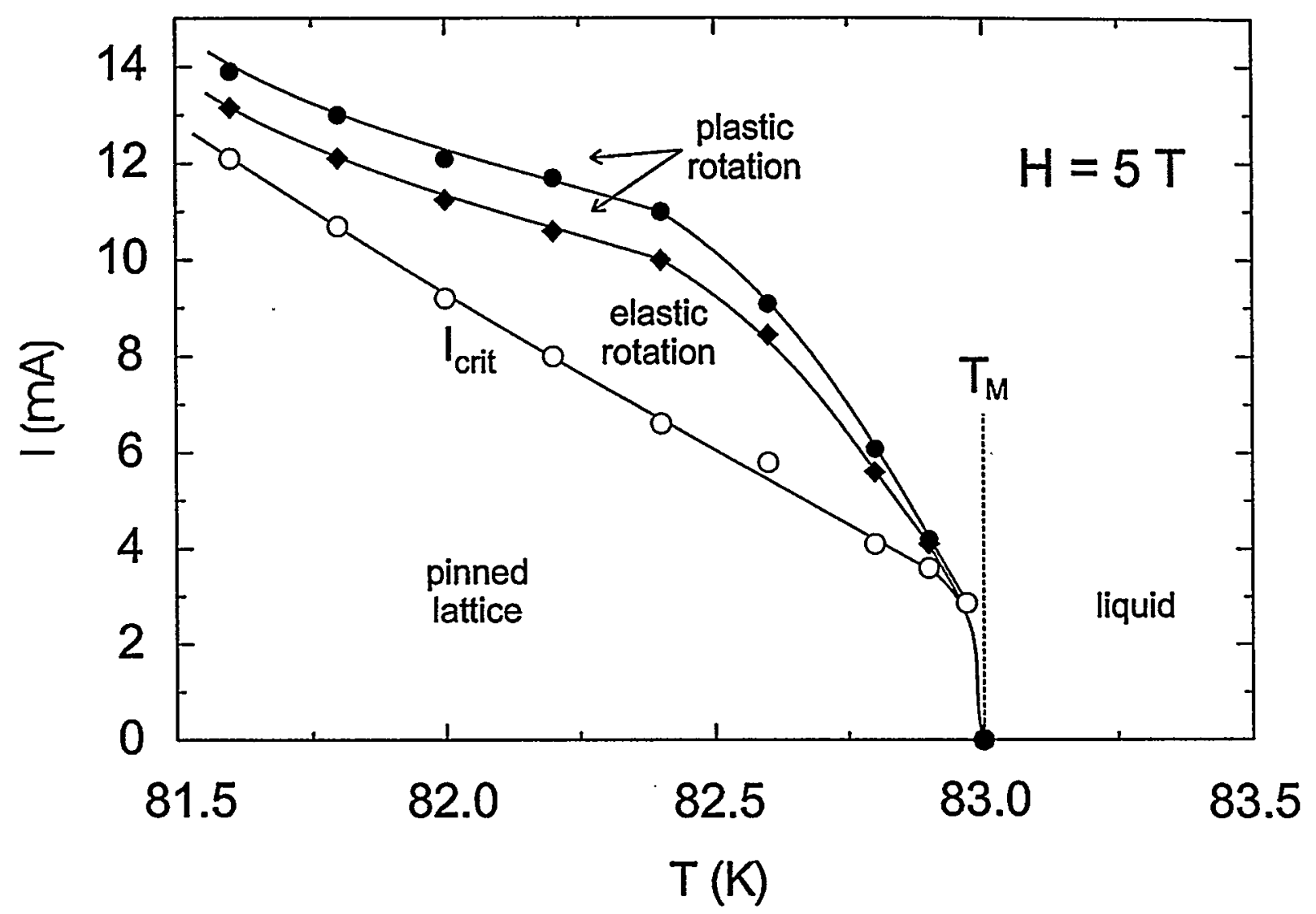

Figure 5

Crabtree ef al 